\title{
Periódicos científicos com indexação descontinuada: a Coleção SciELO Brasil
}

\author{
Scientific journals with discontinued indexing: \\ the SCiELO Brazil Collection
}

\author{
José Paulo Speck PEREIRA' (iD) 0000-0002-6720-9330 \\ Rosângela Schwarz RODRIGUES2 (iD) 0000-0002-9639-6390 \\ Solange Maria dos SANTOS 3 (D) 0000-0002-5067-6362
}

\section{Resumo}

O objetivo do trabalho é identificar os periódicos científicos que tiveram sua indexação descontinuada na Coleção Scientific Electronic Library Online Brasil e analisar as motivações apresentadas nos editoriais. A metodologia consultou as características dos 75 títulos identificados na base e foram analisados os editoriais do ano da desindexação e do posterior, utilizando-se a técnica análise de conteúdo. Os resultados mostram que dos 75 periódicos de todas as áreas, 52 publicaram editoriais sobre o assunto, dos quais retiraram-se 156 textos. A Scientific Electronic Library Online usa duas categorias de motivos genéricos: terminado (40 títulos) - tiveram seus títulos alterados, sendo que 38 permaneceram na base - e indexação interrompida (35 títulos) - que saíram da base e os volumes já indexados foram mantidos. Na categoria terminado, identificaram-se cinco principais motivações: internacionalização e ou visibilidade internacional (62,5\%), modernização (5,0\%), mudança de entidade editora (5,0\%), mudança de entidade editora e internacionalização (2,5\%) e absorvido por outro periódico (2,5\%) - motivações não identificadas (22,5\%). Na categoria indexação interrompida, identificaram-se três principais motivações: internacionalização e ou visibilidade internacional, parceria com editora comercial internacional (34,0\%), atraso no lançamento de novos fascículos $(14,4 \%)$ e contra a política da Scientific Electronic Library Online de internacionalização $(2,9 \%)$ - motivações não identificadas (48,7\%). O grande número de motivações não identificadas em editoriais aponta para a necessidade de ampliar a transparência na gestão dos periódicos. A internacionalização, principal motivação encontrada, é impulsionada pelos critérios da Scientific Electronic Library Online e parece ter sido entendida de várias maneiras nos editoriais. Enquanto a maioria permaneceu na base, foram identificados 12 títulos que firmaram parcerias com editoras comerciais internacionais e saíram da Scientific Electronic Library Online.

Palavras-chave: Bases de dados. Comunicação científica. Indexação. Periódico científico.

\begin{abstract}
The objective of this work was to identify scientific journals that were discontinued in the Scientific Electronic Library Online Brazil Collection and to analyze the motivations expressed in the editorials. The methodology involved identifying the characteristics of the

1 Universidade Federal de Santa Catarina, Centro de Ciências Agrárias, Biblioteca Setorial. Rod. Admar Gonzaga, 1346, Itacorubi, 88034-000, Florianópolis, SC, Brasil. Correspondência para/Correspondence to: J. P. S. PEREIRA. E-mail:<speckpereira@gmail.com>.

2 Universidade Federal de Santa Catarina, Departamento de Ciência da Informação, Programa de Pós-Graduação em Ciência da Informação. Florianópolis, SC, Brasil.

3 Fundação de Amparo à Pesquisa do Estado de São Paulo, Programa Scientific Electronic Library Online. São Paulo, SP, Brasil.

Artigo elaborado a partir da dissertação de J. P. S. PEREIRA, intitulada "Periódicos científicos com indexação descontinuada: a Coleção SciELO Brasil". Universidade Federal de Santa Catarina, 2019

Recebido em 27 de fevereiro de 2020, reapresentado em 24 de outubro de 2020 e aprovado em 29 de outubro de 2020.
\end{abstract}

Como citar este artigo/How to cite this article

Pereira, J. P. S; Rodrigues, R. S.; Santos, S. M. Periódicos científicos com indexação descontinuada: a Coleção SciELO Brasil. Transinformação, v. 32, e200011, 2020. https://doi.org/10.1590/2318-0889202032e200011 
75 journal titles, and the editorials of the year of discontinuation and the subsequent one were analyzed using the content analysis technique. The results show 75 journals from all areas of knowledge, 52 published editorials on the subject, from which 156 texts were identified. Scientific Electronic Library Online uses two categories of generic reasons: finished (40 titles), the titles have been changed, with 38 remaining at the base, and interrupted indexing (35 titles) that were taken off the base, but the volumes already indexed were maintained. In the finished category, five main motivations were identified: internationalization and / or international visibility (62.5\%), modernization (5.0\%), change of publishing entity (5.0\%), change of publishing entity and internationalization (2.5\%), absorbed by another journal (2.5\%), unidentified motivations (22.5\%). In the interrupted indexing category, we identified three main motivations: internationalization and or international visibility, partnership with an international commercial publisher (34.0\%), delay in launching new issues (14.4\%), against Scientific Electronic Library Online internationalization policy (2,9\%), unidentified motivations (48.7\%). The large number of reasons not identified in editorials shows the need to increase transparency in the management of journals. Internationalization, the main motivation found, promoted by Scientific Electronic Library Online criteria and seems to have been understood in several ways in the editorials. While the majority remained at the base, 12 titles that entered into partnerships with international commercial publishers were identified and left Scientific Electronic Library Online.

Keywords: Databases. Scientific research. Indexing. Journal.

\section{Introdução}

A percepção sobre a qualidade da pesquisa se dá principalmente pela publicação dos resultados em periódicos indexados em bases de dados reconhecidas pelo campo do pesquisador. O processo de análise realizado pelos principais indexadores avalia atributos de qualidade do periódico, considerando tanto aspectos intrínsecos (corpo editorial, sistema adotado de revisão por pares, conteúdo científico, entre outros), quanto aspectos extrínsecos (periodicidade e pontualidade de publicação, normalização, instituições mantenedoras, indexadores, indicadores bibliométricos, endogenia, interoperabilidade, entre outros) (Fachin; Medeiros; Rados, 2008; Associação..., 2016; Negahdary, 2017; Passos et al., 2018; International..., 2019).

A indexação propicia ao periódico maior visibilidade e prestígio. Assim, quanto maior o número de bases em que o periódico é indexado, maior o reconhecimento pelas comunidades nacional e internacional. Como consequência, há um potencial aumento da capacidade de atrair manuscritos de qualidade e de citações de outros periódicos (Larivière; Lozano; Gingras, 2014). É o fenômeno conhecido por "efeito Mateus", em que títulos com maior prestígio tendem a receber e publicar os melhores trabalhos, e os periódicos de menor reputação enfrentam mais dificuldades para se manter (Merton, 2013).

A competição por prestígio e visibilidade entre periódicos e pesquisadores revela que a ciência é um campo de disputas. Para Bourdieu (1983, p. 126), o campo é o "lugar de luta política pela dominação científica", cujos pares acumulam capital científico (uma espécie do capital simbólico). O capital científico "[...] consiste no reconhecimento (ou no crédito) atribuído pelo conjunto de pares-concorrentes no interior do campo científico (o número de menções no Citation Index é um bom indicador) [...]" (Bourdieu, 2004, p. 26).

Além das disputas no campo científico, Guédon (2011) destaca que os periódicos científicos tornaramse instrumentos essenciais para regular o sistema internacional de competência científica: as ciências se afirmam universais, o que implica em um sistema de comunicação global que possa regular esse processo, superando regionalismos. A criação de cartéis de editoras comerciais com milhares de títulos e milhões de artigos se desenvolveu ao longo da segunda metade do século XX, com a ampla utilização da base Journal Citation Reports/ Web of Science, da empresa Clarivate, pois seus rankings de citações passaram a definir quais publicações poderiam ser consideradas as principais em cada campo (Guédon, 2011). Nas últimas décadas, houve uma ampliação das cidades e países de origem dos autores produtores de artigos (Csomós, 2018), porém os periódicos de maior prestígio ainda estão concentrados nos grandes centros mundiais, também sede das grandes editoras comerciais.

Nesse cenário, destaca-se a grande contribuição do movimento do Acesso Aberto, ou Open Access (OA), como elemento determinante para o avanço dos periódicos latino-americanos. O sucesso do movimento OA na América Latina explica-se por: (a) historicamente, a região não contou com publishers comerciais e não havia estrutura 
editorial desenvolvida para promover suas publicações que, muitas vezes, já eram distribuídas gratuitamente; (b) adotaram-se ferramentas tecnológicas livres para publicação e disseminação de periódicos e outros trabalhos (como Open Journal Systems e DSpace); (c) comunidades de pesquisadores latinos elaboraram cartas e declarações públicas em favor do OA; (d) criação de indexadores dedicados a promover os periódicos da região como parte do fluxo global de comunicação científica (como Scientific Electronic Library Online [SciELO] e Redalyc); (e) criação de repositórios digitais temáticos ou institucionais; (f) criação de portais de periódicos em universidades (Alperin; Fischman; Willinsky, 2008; Rodrigues; Fachin, 2010; Babini, 2011; Costa; Leite, 2016; Appel; Albagli, 2019;).

No Brasil, a SciELO é o principal indexador de periódicos em OA. Reconhecido por sua capacidade de aumentar a visibilidade dos artigos e conferir prestígio aos periódicos, muitos editores almejam indexar seus títulos nessa base. Uma vez incluídos na SciELO, surge o desafio de competir "[...] nos domínios dos índices internacionais, cujos mecanismos e regras favorecem os periódicos dos países desenvolvidos aí estabelecidos" (Packer, 2014a, p. 304). Para alavancar a qualidade e visibilidade das publicações, os critérios de indexação e permanência na SciELO são rígidos e exigem uma crescente profissionalização das equipes editoriais que mantêm o periódico (Scientific..., 2017).

Todos os anos, alguns periódicos têm sua indexação descontinuada na SciELO, o que pode acarretar prejuízos para a visibilidade das publicações, que passam a não contar mais com a influência e divulgação promovidas pelo indexador. Desde 2014, a SciELO integra o conjunto de bases da Web of Science sob o nome de SciELO Citation Index (Packer, 2014b). Alguns comitês de avaliação da Capes Qualis têm a indexação na SciELO como um dos parâmetros para classificação nos estratos mais altos A1, A2, A3, ou A4, a exemplo das áreas de História, Educação, Antropologia e Arqueologia (Coordenação..., 2019a, 2019b, 2019c). Além disso, a presença na SciELO é um dos critérios para a participação em editais de financiamento de periódicos (Conselho..., 2019).

Devido à importância da indexação de periódicos para a comunidade acadêmica, o presente trabalho tem por objetivo identificar os periódicos científicos que tiveram sua indexação descontinuada na Coleção SciELO Brasil e analisar as motivações apresentadas nos editoriais que os periódicos publicaram logo após a saída da base.

Ao abordar a interface entre periódicos científicos e indexadores, a pesquisa se insere nos estudos sobre comunicação científica da ciência da informação. Periódicos científicos são um dos objetos de estudo mais investigados na área, especialmente pela condição de ser a parte mais visível de uma pesquisa e unidade de avaliação de desempenho de pesquisadores e instituições (Gingras, 2020). Krauskopf (2018) analisou periódicos com indexação descontinuada na Scopus, verificando que os sites oficiais dos títulos ainda mencionavam a indexação na base. Trabalhos dessa natureza revelam contextos de produção dos periódicos e contribuem para o aumento da transparência das publicações.

\section{Procedimentos Metodológicos}

O corpus de análise constituiu-se dos periódicos com indexação descontinuada (coleção não-corrente) da Coleção SciELO Brasil, a maior e mais antiga base da plataforma SciELO. Os dados analisados foram: datas de admissão e saída da base; motivo oficial da saída da base; área temática categorizada pela SciELO; entidade editora - expressão adotada por Mueller (2011) -, idiomas de publicação e idioma da grafia dos títulos novos, e, por fim, periodicidade. Os dados foram tabulados em planilha eletrônica e disponibilizados no repositório Zenodo (Pereira; Rodrigues; Santos, 2020).

Adotou-se o termo indexação descontinuada porque os periódicos que saíram da base continuaram a lançar novos fascículos em outras plataformas, mas os volumes já indexados permaneceram disponíveis para consulta. $O$ termo 'descontinuado' aparece nas diretrizes da SciELO “[...] a indexação é descontinuada quando o periódico deixa de publicar durante 6 meses" (Scientific..., 2017, p. 15). 
As datas de admissão e saída da base são as indicadas no site que cada publicação possui na SciELO Brasil. Na maioria dos casos, as datas correspondem aos anos efetivos de mudanças, mas há exceções, e a principal delas trata-se do Boletim do Instituto Paulista de Oceanografia, que em 1952 teve seu título alterado para Boletim do Instituto Oceanográfico. Em 1996 mudou novamente para Revista Brasileira de Oceanografia, e em 2004 foi novamente substituído, dessa vez para Brazilian Journal of Oceanography. Apesar das primeiras mudanças terem ocorrido anteriormente à criação da SciELO, elas foram computadas devido ao acervo dos títulos estarem indexados na base. Cada alteração de título foi contabilizada como um novo periódico.

Para obter mais detalhes sobre os motivos da saída da base, foram analisados os editoriais publicados no último ano ainda na SciELO e do ano posterior, logo após a indexação ter sido descontinuada. Com o objetivo de analisar os editoriais foi utilizada a técnica de análise de conteúdo (Bardin, 1979; Fonseca Junior, 2012). O cruzamento entre as análises dos editoriais e os dados do periódico coletados permitiu a identificação das motivações que levaram à descontinuidade da indexação.

\section{Resultados e Discussão}

A Coleção SciELO Brasil possuía, em julho 2018, 365 periódicos indexados, sendo 290 com indexação corrente e 75 com indexação descontinuada. O corpus da pesquisa constituiu-se dos 75 periódicos científicos com indexação descontinuada, sendo que a base indica duas categorias de motivos para a saída das publicações: (a) Categoria terminado: 40 títulos. Tiveram seus títulos alterados, às vezes mais de uma vez, sendo que 38 permaneceram na base e (b) Categoria indexação interrompida: 35 títulos. Saíram da base e os volumes indexados foram mantidos. Entre os 75 periódicos, 52 publicaram editoriais sobre o assunto, dos quais retiraram-se 156 textos (115 da categoria Terminado e 41 da categoria Indexação Interrompida).

As motivações que levaram os periódicos a ter a indexação descontinuada foram identificadas a partir da análise de conteúdo dos editoriais, em cruzamento com os dados sobre entidades editoras, periodicidades, idiomas de publicação e idioma da grafia dos títulos novos e datas de admissão e saída da SciELO. A Tabela 1 apresenta a categoria terminado, com 40 títulos no total.

Dos 40 periódicos da categoria terminado, 38 permaneceram indexados na SciELO com novos títulos e a internacionalização e ou visibilidade internacional foi o motivo mais frequente $(62,5 \%)$. Todas as publicações dessa categoria, ao manterem-se na SciELO, reiteraram o compromisso com o acesso aberto. Somente a RAE Eletrônica e a Entomología y Vectores saíram em definitivo. O primeiro periódico, porque foi absorvido por outra publicação. Já o segundo não foi localizado na web após a saída da base e não havia indicação de mudança de título.

Pertence às Ciências da Saúde a maioria dos títulos descontinuados (42,5\%), seguido das Ciências Biológicas $(17,5 \%)$ e títulos categorizados em mais de uma área na SciELO (15,0\%). Historicamente, as Ciências da Saúde destacam-se na Coleção SciELO Brasil, com grande volume de títulos indexados, o que pode ser explicado pela parceria com a Bireme na fundação da SciELO. No entanto, a diversidade de áreas temáticas descritas na Tabela 1 confirma a cobertura ampla da base.

Há poucos periódicos das Ciências Sociais Aplicadas (7,5\%) e Humanas (5,0\%) dentre aqueles que mudaram de título, com pouca participação na categoria "Internacionalização" (Tabela 1). Os resultados vão ao encontro do trabalho de Mugnaini, Digiampetri e Mena-Chalco (2014), que analisou a produção brasileira de artigos indexados na Web of Science e SciELO no período 1998-2012, e constatou que algumas áreas são mais internacionalizadas que outras, além de que as ciências sociais e humanidades publicavam predominantemente em títulos nacionais nas Zonas de Bradford 1 e 2. Somente na Zona 3 aparecem artigos publicados em títulos estrangeiros. 
Tabela 1. Motivações para a saída dos periódicos da Coleção SciELO Brasil (categoria Terminado), agrupados por áreas temáticas.

\begin{tabular}{|c|c|c|c|}
\hline \multirow{2}{*}{ Motivos } & \multirow{2}{*}{ Áreas } & \multicolumn{2}{|c|}{ Títulos } \\
\hline & & $n$ & $\%$ \\
\hline \multirow[t]{7}{*}{ Internacionalização e ou visibilidade internacional } & Saúde & 12 & 30,0 \\
\hline & Biológicas & 4 & 10,0 \\
\hline & Agrárias & 3 & 7,5 \\
\hline & Engenharia & 2 & 5,0 \\
\hline & Inserido em mais de uma área & 2 & 5,0 \\
\hline & Sociais Aplicadas & 1 & 2,5 \\
\hline & Humanas & 1 & 2,5 \\
\hline Total & & 25 & 62,5 \\
\hline \multirow[t]{2}{*}{ Modernização } & Humanas & 1 & 2,5 \\
\hline & Inserido em mais de uma área & 1 & 2,5 \\
\hline Total & & 2 & 5,0 \\
\hline \multirow[t]{2}{*}{ Mudança de entidade editora } & Saúde & 1 & 2,5 \\
\hline & Inserido em mais de uma área & 1 & 2,5 \\
\hline Total & & 2 & 5,0 \\
\hline Mudança de entidade editora e internacionalização & Saúde & 1 & 2,5 \\
\hline Total & & 1 & 2,5 \\
\hline Absorvido por outro periódico & Sociais Aplicadas & 1 & 2,5 \\
\hline Total & & 1 & 2,5 \\
\hline \multirow[t]{4}{*}{ Não identificados } & Biológicas & 3 & 7,5 \\
\hline & Saúde & 3 & 7,5 \\
\hline & Inserido em mais de uma área & 2 & 5,0 \\
\hline & Sociais Aplicadas & 1 & 2,5 \\
\hline Total & & 9 & 22,5 \\
\hline Total & & 40 & 100,0 \\
\hline
\end{tabular}

Fonte: Elaborado pelos autores (2019), dados coletados em julho de 2018.

Nota: SciELO: Scientific Electronic Library Online.

A primeira motivação identificada nos editoriais publicados foi Internacionalização e/ou Visibilidade internacional, que agrupa 25 publicações (62,5\%). Embora essa motivação vá ao encontro das atuais diretrizes da SciELO Brasil e de suas linhas prioritárias de ação, que buscam promover a profissionalização, internacionalização e sustentabilidade das publicações (Packer; Meneghini, 2014; Scientific..., 2017), é importante observar que no caso desses títulos a busca por internacionalização é anterior à publicação das diretrizes da SciELO de 2014 (Scientific..., 2014), que passaram declaradamente a incentivá-la. Em 14 editoriais foram registradas motivações e expectativas declaradas pelos editores relacionadas à internacionalização (Quadro 1). Cada periódico expressa seus objetivos quanto à internacionalização e, de forma geral, todos buscam projeção internacional. Algumas publicações desejavam desvencilhar-se da imagem de publicação regional, limitada às questões nacionais, e competir em um 'mercado' mais amplo, de modo a ser um canal de comunicação representativo além das fronteiras de seu país. Os editores esperam que os títulos projetem a ciência brasileira para o mundo. Desejam, também, que pesquisadores de outros países passem a enviar seus manuscritos para publicação.

Os periódicos ditos nacionais, que rompem com os limites de seu entorno e se internacionalizam, deixam de ser identificados por seu país de publicação, ou instituição editora, e assumem seu papel no circuito internacional de circulação do conhecimento (Beigel, 2014). Por essa razão, Santos e Mugnaini (2019) destacam que a divisão entre periódicos nacionais ou internacionais passa a se basear muito mais na cobertura deste circuito, sendo o país de publicação de um título importante muito mais para a comunidade científica nacional do que para o resto do mundo. 
Quadro 1. Motivações da internacionalização expressas nos editoriais publicados pelos periódicos que saíram da Coleção SciELO Brasil (categoria Terminado).

\begin{tabular}{|c|c|c|}
\hline Áreas & Motivações & Título antigo/Título novo \\
\hline \multirow{5}{*}{ 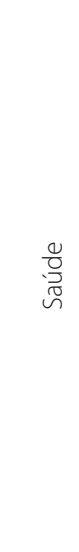 } & Expectativa que o título se torne o principal da América Latina em seu & Arquivos Internacionais de Otorrinolaringologia/ \\
\hline & $\begin{array}{l}\text { Aumentar a visibilidade e trazer autores internacionais para o periódico } \\
\text { (Behlau, 2012). }\end{array}$ & Jornal da Sociedade Brasileira de Fonoaudiologia/CoDAS \\
\hline & $\begin{array}{l}\text { Ampliar a visibilidade, indexação em bases e aumentar o fator de impacto } \\
\text { (FI), internacionalização passa pela publicação em língua inglesa (Abdalla, } \\
\text { 2008; Ferreira, 2009). }\end{array}$ & $\begin{array}{l}\text { Revista Brasileira de Ciências Farmacêuticas/Brazilian } \\
\text { Journal of Pharmaceutical Sciences }\end{array}$ \\
\hline & $\begin{array}{l}\text { Visibilidade internacional. Atingir a classificação A1 do sistema Qualis/ } \\
\text { Capes. Ser incluída em outros importantes indexadores, principalmente } \\
\text { Medline e Institute for Scientific Information (Web of Science). O título está } \\
\text { pronto para contribuir com o corpo de conhecimento internacional } \\
\text { produzido no campo da psiquiatria e da psicoterapia (Kapczinski, 2011a, } \\
2011 \text { b). }\end{array}$ & $\begin{array}{l}\text { Revista de Psiquiatria do Rio Grande do Sul/Trends in } \\
\text { Psychiatry and Psychotherapy }\end{array}$ \\
\hline & Que o periódico tenha impacto internacional. (Rocha-e-Silva, 2005). & Revista do Hospital das Clínicas/Clinics \\
\hline \multirow{3}{*}{$\begin{array}{l}\frac{\widetilde{U}}{\bar{T}} \\
\frac{0}{0} \\
\frac{0}{\infty}\end{array}$} & $\begin{array}{l}\text { Projeção internacional (Martinez, 2001). } \\
\text { Consolidação como canal de comunicação representativo da região } \\
\text { (Martinez, 2001). }\end{array}$ & $\begin{array}{l}\text { Anais da Sociedade Entomológica do Brasil/Neotropical } \\
\text { Entomology }\end{array}$ \\
\hline & $\begin{array}{l}\text { Busca pela internacionalização da ciência brasileira e regional (Moura } \\
\text { Duarte, 1998). }\end{array}$ & $\begin{array}{l}\text { Brazilian Journal of Genetics/Genetics and Molecular } \\
\text { Biology }\end{array}$ \\
\hline & $\begin{array}{l}\text { Indexação no Institute for Scientific Information (Web of Science) (Editorial } \\
\text { Note, 2002). }\end{array}$ & Revista Brasileira de Biologia/Brazilian Journal of Biology \\
\hline \multirow{3}{*}{$\frac{\sqrt{0}}{\frac{\pi}{\sqrt[0]{0}}}$} & Penetração nacional e internacional (Reichardt,1991, 1992). & $\begin{array}{l}\text { Anais da Escola Superior de Agricultura Luiz de Queiroz/ } \\
\text { Scientia Agricola }\end{array}$ \\
\hline & $\begin{array}{l}\text { O Brasil tem vocação para liderar a busca de conhecimento no campo da } \\
\text { agricultura (Pfenning et al., 2008). }\end{array}$ & Fitopatologia Brasileira/Tropical Plant Pathology \\
\hline & Visibilidade e aumento do fator de impacto (Pfenninget al., 2008). & \\
\hline 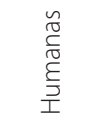 & $\begin{array}{l}\text { Melhor difusão dos conhecimentos da área educacional, estimular } \\
\text { o debate acadêmico e favorecer um maior intercâmbio entre os } \\
\text { especialistas nacionais e estrangeiros (Bueno, 1999). }\end{array}$ & Revista da Faculdade de Educação/Educação e Pesquisa \\
\hline
\end{tabular}

Fonte: Elaborado pelos autores (2019).

Nota: Capes: Coordenação de Aperfeiçoamento de Pessoal de Nível Superior; SciELO: Scientific Electronic Library Online.

No Brasil, o debate sobre a internacionalização de periódicos perpassa o sistema Qualis de avaliação de periódicos da Capes. Os critérios Qualis de 2019 enfatizaram o uso das métricas CiteScore (base Scopus-Elsevier), Fator de Impacto (FI) (base Web of Science - Clarivate) e o H5 (base Google Scholar) para todas as áreas, obrigando as comissões de avaliação a adaptarem-se. Para as áreas de Medicina I, por exemplo, possuir Fl ou CiteScore acima de 3 é condição para alcançar os estratos mais elevados. Para os periódicos de Educação, as métricas são analisadas junto a outros critérios (Coordenação..., 2019b, 2019d).

Pires et al. (2020), ao analisarem a influência do Qualis na veiculação da produção científica brasileira entre 2007 e 2016, esclarecem que a necessidade de publicar em títulos classificados no Qualis faz com que muitos pesquisadores selecionem aqueles com menor dificuldade de submissão:

Dessa forma, percebe-se que a lista Qualis pode estar direcionando a escolha de periódicos para um subconjunto com baixo ou nenhum impacto internacional, na medida em que a classificação Qualis destes periódicos pode garantir ao pesquisador a concessão ou manutenção de um determinado recurso financeiro ou benefício (Pires et al., 2020, p. 13).

O fato de 62,5\% dos periódicos terem seus títulos alterados visando à internacionalização (Tabela 1) indica um movimento em resposta à classificação do Qualis de integração à ciência mundial e obtenção de maior visibilidade. 
A segunda motivação identificada, "Modernização", agrupa duas publicações cujos editoriais indicaram a necessidade de atualização frente aos novos tempos. São eles: Boletim do Instituto Oceanográfico, que teve seu título alterado, em 1996, para Revista Brasileira de Oceanografia, e Revista do Departamento de Psicologia - UFF, cujo título foi modificado - em 2008, para Fractal: Revista de Psicologia. O editorial do Boletim do Instituto Oceanográfico registra que: "[...] irá surgir uma nova publicação, mais abrangente, moderna e atual, a Revista Brasileira de Oceanografia (Brazilian Journal of Oceanography) [...]" (Paiva Filho, 1995, não paginado). No editorial da Revista do Departamento de Psicologia - UFF foi dado especial destaque à opção pelo formato eletrônico de publicação, além das expectativas com a mudança de título: "Trata-se para nós de um momento histórico que é ao mesmo tempo, uma celebração do percurso que trilhamos até este momento e uma abertura de outros caminhos a trilhar" (Moraes; Nascimento, 2008, não paginado). O relato mostra que em 2008 o formato digital ainda parecia um desafio para os editores e revistas, tornando-se obrigatório após poucos anos, sendo que os títulos que ainda publicam uma versão impressa são exceções.

A terceira motivação identificada, "Mudança de entidade editora", agrupa duas publicações que foram transferidas para outras entidades editoras. O Boletim do Instituto Paulista de Oceanografía recebeu o título de Boletim do Instituto Oceanográfico e Pró-Fono Revista de Atualização foi alterada para Jornal da Sociedade Brasileira de Fonoaudiologia. Os títulos antigos faziam menção às entidades anteriores, por isso foi necessário que as novas instituições os rebatizassem, mas a menção à entidade editora foi mantida. Anos depois, as duas publicações tiveram novamente seus títulos alterados, mas dessa vez as palavras indicavam apenas as áreas temáticas.

O Boletim do Instituto Paulista de Oceanografia antecedeu o Boletim do Instituto Oceanográfico. A mudança ocorreu em 1952 em virtude de o Instituto ter sido transferido para a Universidade de São Paulo (Advertência..., 1952). Nas décadas seguintes, a publicação ainda alteraria seu título para Revista Brasileira de Oceanografia (em 1996) e Brazilian Journal of Oceanography, em 2004.

No caso da Pró-Fono Revista de Atualização Científica, a mudança ocorreu em 2011, quando a empresa Pró-Fono transferiu seu periódico para a Sociedade Brasileira de Fonoaudiologia. A partir de então, mudou-se o título para Jornal da Sociedade Brasileira de Fonoaudiologia. Em 2013, esta publicação teve seu título novamente alterado para Communication Disorders, Audiology and Swallowing (CODAS).

A quarta motivação identificada engloba a"Mudança de entidade editora e internacionalização", e representa o periódico Revista da Sociedade Brasileira de Fonoaudiologia, que teve seu título alterado para Audiology: Communication Research. Essa publicação pertencia à Sociedade Brasileira de Fonoaudiologia, a mesma instituição que administrava o Jornal da Sociedade Brasileira de Fonoaudiologia, mencionado anteriormente. Essa instituição optou por editar apenas uma publicação, o Jornal, sendo que a Revista foi transferida para a Academia Brasileira de Audiologia, que alterou o título para Audiology: Communication Research com o objetivo de, entre outras mudanças, promover sua internacionalização.

A quinta motivação identificada, "Absorvido por outro periódico", representa o periódico RAE Eletrônica. Consta em seus editoriais que ele foi absorvido por outra publicação, a Revista de Administração de Empresas (RAE), cuja mudança veio acompanhada do aumento da periodicidade de trimestral para bimestral. Ainda na categoria terminado, não foi possível identificar informações sobre as motivações da indexação descontinuada de nove periódicos. Nenhum deles publicou editoriais no ano do desligamento da SciELO Brasil ou no ano posterior.

A Coleção SciELO Brasil apresenta a categoria indexação interrompida, com 35 títulos, constituída por aqueles que saíram da base, cujos motivos identificados são apresentados na Tabela 2. Há títulos de todas as áreas do saber dentre aqueles que saíram da SciELO, com destaque para as Ciências Humanas (23\%), que tiveram a indexação descontinuada devido ao atraso no lançamento de novos fascículos, ou por serem contra a política da SciELO de internacionalização ou, ainda, por outros motivos não identificados. Em seguida, estão as Ciências da Saúde (20\%) e Ciências Exatas e da Terra (17\%), sendo que estas últimas concentram mais títulos dentre aqueles que saíram visando à internacionalização. Os títulos desta categoria pertencem predominantemente às chamadas ciências da natureza ou "ciências duras". 
Tabela 2. Motivações para a saída dos periódicos da Coleção SciELO Brasil (categoria Indexação interrompida), agrupados por áreas temáticas.

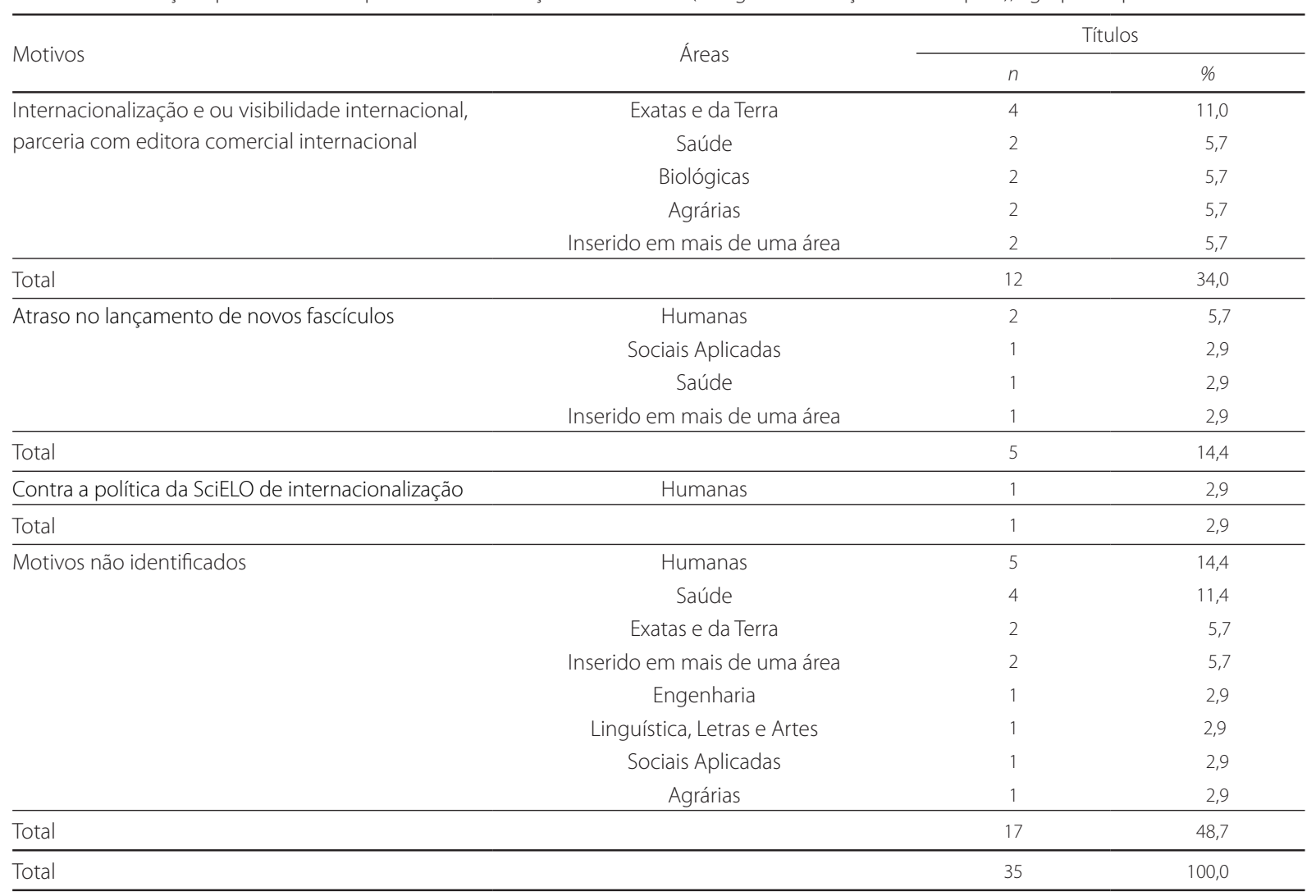

Fonte: Elaborado pelos autores (2019), dados coletados em julho de 2018.

Nota: Valores percentuais arredondados. SciELO: Scientific Electronic Library Online.

A motivação com maior incidência (34\%) foi (Internacionalização e/ou visibilidade internacional, parceria com editora comercial internacional), que agrupa 12 periódicos que se associaram a editoras comerciais internacionais, optando pelo acesso por subscrição (leitores pagam para acessar os artigos), o que os obrigou a sair da base, que somente aceita títulos em acesso aberto. A distribuição de títulos entre as editoras comerciais foi a seguinte: Springer com nove títulos; Elsevier com dois títulos e American Psychological Association (APA) com um título.

Ainda sobre esse tema, Xu et al. (2019) analisaram estratégias de internacionalização de 435 periódicos científicos chineses e identificaram como sendo as mais relevantes: (a) a inclusão de pesquisadores importantes de outros países no conselho editorial; (b) visibilidade online; (c) conversão de periódico nacional em internacional; e (d) o estabelecimento de parcerias com editoras comerciais internacionais. Entre as editoras comerciais operando em parceria com os periódicos chineses, os pesquisadores identificaram que as três maiores eram: Springer (113 títulos), Elsevier (73) e Wiley (14), sendo que as duas primeiras também predominaram entre os periódicos brasileiros que se associaram a editoras comerciais.

Lin e Zhan (2016) argumentam que muitos dos títulos chineses em inglês consideram limitados os benefícios da parceria com grandes editoras comerciais, provavelmente por conta das expectativas pouco realistas e por julgarem que os custos da parceria seriam melhor investidos na obtenção de artigos de alta qualidade.

De acordo com Pozzobon e Camargo (2019), editoras comerciais têm publicado periódicos também no Brasil, mas em escala muito menor. Os autores identificaram 134 títulos brasileiros na base Web of Science, dos quais nove (6,71\%) eram publicados pelas editoras internacionais Springer (7 títulos) e Elsevier (2). Vendo com preocupação o 
crescimento das editoras comerciais no país, os pesquisadores ressaltam a importância da manutenção do acesso aberto para promover a ciência e questionam a comunicação científica sob a lógica do capital.

Em nove editoriais foram registradas as motivações e expectativas dos editores relacionadas à internacionalização e às mudanças que ocorreram no período de saída da Coleção SciELO Brasil, sendo a parceria com as editoras considerada um dos meios de atingir seus objetivos (Quadro 2). O título Psychology \& Neuroscience foi o único nesta categoria a mencionar explicitamente o desligamento da Coleção SciELO Brasil. No ano seguinte, já fora da SciELO, a APA assumiu sua publicação. Landeira-Fernandez et al. $(2015$, p.1) detalham os serviços que a entidade editora oferecerá:

During this period, authors will submit their manuscripts to the Editorial Manager platform online. APA will support and manage the electronic platform system, and the Editors will conduct the electronic peer-review process. Once accepted, the manuscript will be forwarded to the APA production office where it will be formatted according to the APA style, including

Quadro 2. Motivações da internacionalização expressas nos editoriais publicados pelos periódicos que saíram da Coleção SciELO Brasil (categoria Indexação interrompida).

\begin{tabular}{|c|c|c|c|}
\hline Áreas & Motivações & Título & Entidade editora \\
\hline 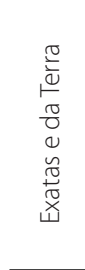 & $\begin{array}{l}\text { Visibilidade internacional e disputar com as publicações de } \\
\text { prestígio da área (Oliveira; Breitman, 2009). } \\
\text { Com a mudança de título, o periódico tornou-se "verda- } \\
\text { deiramente internacional"(Silva, 2013, p.1, tradução nossa) } \\
\text { (SBA Controle \& Automação teve seu título alterado para } \\
\text { Journal of Control, Automation and Electrical Systems quando } \\
\text { saiu da SciELO, passando a ser publicada pela Springer). }\end{array}$ & $\begin{array}{l}\text { Journal of Control, Automation } \\
\text { and Electrical Systems }\end{array}$ & $\begin{array}{l}\text { Sociedade Brasileira de Automá- } \\
\text { tica/Springer }\end{array}$ \\
\hline \multirow[b]{2}{*}{$\frac{1}{0}$} & $\begin{array}{l}\text { Aumento da visibilidade internacional (Camargo et al., } \\
\text { 2016). }\end{array}$ & Brazilian Journal of Physical Therapy & $\begin{array}{l}\text { Associação Brasileira de Pesquisa } \\
\text { e Pós-Graduação em Fisioterapia/ } \\
\text { Elsevier }\end{array}$ \\
\hline & $\begin{array}{l}\text { Benefícios da parceria com Elvesier: padrões gráficos da } \\
\text { editora, exposição proporcionada pela presença no Science } \\
\text { Direct, expansão do conselho editorial internacional, } \\
\text { adoção de sistema gerenciador de publicações (Chaves, } \\
\text { 2014, 2015). }\end{array}$ & $\begin{array}{l}\text { Revista Brasileira de Cardiologia } \\
\text { Invasiva }\end{array}$ & $\begin{array}{l}\text { Sociedade Brasileira de Hemodi- } \\
\text { nâmica e Cardiologia Interven- } \\
\text { cionista/Elsevier }\end{array}$ \\
\hline \multirow{2}{*}{$\begin{array}{l}\frac{n}{0} \\
\frac{.}{\sigma} \\
\frac{O}{0} \\
\frac{0}{\infty}\end{array}$} & $\begin{array}{l}\text { Aumento na submissão de artigos por pesquisadores de } \\
\text { diversos países (Salatino, 2012). }\end{array}$ & Brazilian Journal of Botany & $\begin{array}{l}\text { Sociedade Botânica de São Paulo/ } \\
\text { Springer }\end{array}$ \\
\hline & $\begin{array}{l}\text { Renome e reconhecimento internacional na área (Cômsoli, } \\
\text { 2012). }\end{array}$ & Neotropical Entomology & $\begin{array}{l}\text { Sociedade Entomológica do Brasil/ } \\
\text { Springer }\end{array}$ \\
\hline$\frac{\sqrt[2]{0}}{\frac{\pi}{\frac{\pi}{0}}}$ & $\begin{array}{l}\text { Mudou o título de Brazilian Journal of Plant Physiology } \\
\text { para Theoretical and Experimental Plant Physiology. Retirada } \\
\text { a palavra "brazilian" para ampliar o acesso e incentivar } \\
\text { pesquisadores de outros países a publicar bons estudos da } \\
\text { área (Bressan-Smith, 2013). }\end{array}$ & $\begin{array}{l}\text { Theoretical and Experimental Plant } \\
\text { Physiology }\end{array}$ & $\begin{array}{l}\text { Sociedade Brasileira de Fisiologia } \\
\text { Vegetal/Springer }\end{array}$ \\
\hline 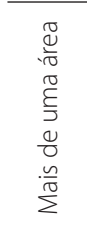 & $\begin{array}{l}\text { Indexação em mais bases de dados para aumentar a } \\
\text { visibilidade, como Medline e Science Citation Index; melhorar } \\
\text { a avaliação na Qualis Capes; expansão do conselho editorial; } \\
\text { alcançar mais independência e sustentabilidade através } \\
\text { da parceria com uma editora acadêmica ou sociedade } \\
\text { cientifica (Mograbi, 2014). }\end{array}$ & Psychology \& Neuroscience & $\begin{array}{l}\text { Instituto Brasileiro de Neuropsico- } \\
\text { logia e Comportamento/American } \\
\text { PsychologicalAssociation }\end{array}$ \\
\hline
\end{tabular}

Fonte: Elaborado pelos autores (2019).

Nota: Capes: Coordenação de Aperfeiçoamento de Pessoal de Nível Superior; SciELO: Scientific Electronic Library Online.

$\overline{4}$ No original: "[...] truly international" (Silva, 2013, p. 1). 
technical editing and proofreading services. Electronic proofs will be provided to the authors for review. APA will also be responsible for all corrections to the page proofs. Once the production is completed, the paper will be published Online First (also known as published ahead of print or preissue publishing) and then appear in one of the four issues published annually (Landeira-Fernandez et al., 2015, p. 1).

O Instituto Brasileiro de Neuropsicologia e Comportamento continuou a ser o proprietário de Psychology \& Neuroscience e detentor do seu controle editorial, mas cedeu à APA o direito de distribuição e comercialização pelos sete anos seguintes. (Landeira-Fernandez et al., 2015).

Dentro de um campo científico, a autoridade de um agente, "[...] que assegura um poder sobre os mecanismos constitutivos de campo [...]" (Bourdieu, 1983, p. 127), é obtida por meio de disputas e acumulação de capital científico. Entende-se que a parceria com as editoras comerciais internacionais se configura como uma estratégia de obtenção de capital científico, pela transmissão, aos periódicos, do capital dessas entidades.

Nas situações apresentadas, podem-se estabelecer comparações entre as avaliações Qualis Periódicos e o comportamento apresentado pelos títulos brasileiros que almejam a internacionalização. Schifini e Rodrigues (2019) analisaram 528 títulos classificados como A1 nas áreas de Medicina I, II e III e constataram que não existiam títulos brasileiros nesse estrato, pois nenhum título nacional possuía as métricas Fl acima de 4 ou citações por documento exigidas. A melhor classificação possível dos títulos brasileiros nessas áreas seria B2 e um título indexado na SciELO seria B4. A maioria dos títulos A1 provinha dos Estados Unidos (44,5\%), do Reino Unido (38,25\%), da Holanda (6,43\%) e da Alemanha (3,97\%), totalizando 93,15\%, sendo que os 6,85\% títulos restantes eram oriundos de outros dez países. Ao reunir as editoras de um mesmo grupo editorial, constataram que "[...] mais da metade dos periódicos A1 das áreas de medicina do Qualis são editorados por 3 grandes grupos: Elsevier, Wiley e Springer Nature" (Schifini; Rodrigues, 2019, p. 97).

Em análise similar, Fernandes e Manchini (2019) investigaram os títulos dos estratos A1 e A2 das áreas de Economia, Administração, Ciências Contábeis e Turismo. Constataram que não há títulos brasileiros nos estratos A1 dessas áreas, sendo todos predominantemente dos Estados Unidos, Reino Unido e Países Baixos. A partir dos estratos A2 o Brasil começa a ter presença tímida: 2,4\% nos títulos de Economia e 9,6\% nas demais áreas. Esses resultados reforçam a ideia de que os estratos superiores do Qualis privilegiam títulos estrangeiros com alto fator de impacto oriundos de países desenvolvidos, sedes de grandes editoras comerciais internacionais: Estados Unidos (Wiley-Blackwell e Sage), Reino Unido (Taylor \& Francis), Holanda (Elsevier) e Alemanha (Springer) (Schifini; Rodrigues, 2019).

Como já mencionado, as classificações Qualis podem ter estimulado pesquisadores a publicar em títulos estrangeiros, mesmo que tenham pouco ou nenhum impacto internacional (Pires et al., 2020). Nesse cenário, é necessário debater os possíveis impactos negativos do Qualis sobre os periódicos editados no Brasil, assim como, sobre o movimento do acesso aberto brasileiro à informação científica. Se os estratos mais altos, em muitas áreas, são povoados por títulos estrangeiros de acesso restrito publicados por editoras comerciais, a mensagem aos pesquisadores é para que enviem seus melhores trabalhos aos periódicos estrangeiros de acesso restrito. Desse modo, se por um lado há um esforço brasileiro de criar uma infraestrutura de pesquisa que busca desenvolver capacidade nacional de publicar periódicos de qualidade, por outro lado há o estímulo na direção contrária: não se valoriza na mesma proporção a publicação em periódicos de qualidade do Brasil, os quais, relegados a posições secundárias de classificação, são vistos pelos pesquisadores como pouco atrativos para serem considerados a primeira opção de publicação de seus melhores artigos. Nesse contexto de hipervalorização das métricas da ciência, parece lógico que um editor suponha que seria mais fácil para um título brasileiro alcançar indexação, obter fator de impacto e enquadrar-se como A1 se estiver vinculado a uma editora comercial de prestígio internacional. 
Outro aspecto importante a ser levado em conta sobre os periódicos de OA é a sustentabilidade financeira. Em sua pesquisa sobre financiamento de periódicos indexados na SciELO, Mueller (2011) aponta a existência de fontes internas e externas de financiamento. As primeiras referem-se a: custos de publicação (taxa cobrada do autor), assinaturas e venda avulsa. A cobrança de taxas continua a ser adotada por muitos periódicos em OA, ao passo que as assinaturas ou venda avulsa pertencem à época da distribuição de edições impressas. Quantos às fontes externas, são elas: CNPq/Capes, fundações de amparo estaduais, entidades comerciais, entre outras.

Appel e Albagli (2019) analisaram 81 títulos brasileiros OA identificados da base DOAJ que cobram Article Processing Charge (APC). Explicam que o preço médio do APC é de 920BRL 265USD, mas há variação entre as áreas, e mais de $50 \%$ dos títulos provêm de universidades. O estudo revela que quanto maior o estrato Qualis (A1, A2, B1), maior é a taxa de APC cobrada. Não foram encontradas relações significativas entre o valor do APC e as métricas FI e Scimago Journal Rank, além de apenas uma relação moderada com o Índice h da Scimago. O trabalho de Appel e Albagli (2019) reforça o entendimento de que as decisões gerenciais dos periódicos científicos brasileiros, mantidos por universidades em sua maioria, estão bastante alinhadas à classificação Qualis, que compõe parte do sistema de avaliação dos programas de pós-graduação brasileiros.

Ao longo das últimas décadas, observou-se que diversos periódicos substituíram o clássico modelo de subscrição por uma variedade de modelos de acesso livre ou híbridos, impulsionados pelo impacto do movimento OA. Matthias, Jahn e Laakso (2019) explicam, no entanto, que o movimento contrário também ocorre. Os autores designam por reverse flip, aqui traduzido por "conversão contrária", o fenômeno em que periódicos abandonam o modelo OA e migram para formas restritivas de acesso. Na presente pesquisa, pode-se afirmar que os 12 títulos que foram desligados da SciELO porque firmaram parcerias com editoras comerciais são casos de conversão contrária.

A análise de uma dessas publicações sugere que as decisões sobre pagamento de subscrição ou OA não são permanentes. Como exemplo, tem-se o periódico Journal of the Brazilian Computer Society, que teve a indexação descontinuada em 2010 pelo motivo indexação interrompida. A Sociedade Brasileira de Computação informa em seu site que o título passou a ser publicado pela Springer a partir de 2010 em versão impressa e seus associados teriam acesso online pela plataforma da editora. Mas a partir de 2014 o periódico passou a ser OA novamente, disponível na plataforma Springer Open.

A segunda motivação identificada de descontinuidade da indexação foi "Atraso no lançamento de novos fascículos", que agrupa cinco títulos. Desses, somente dois títulos mencionaram explicitamente o desligamento da SciELO Brasil em virtude do atraso no lançamento de novos fascículos: Estudos de Psicologia (Natal) e Psicologia em Estudo. O editor de Psicologia em Estudo, ao explicar os motivos do desligamento da Coleção SciELO Brasil, faz uma correlação com aspectos das conjunturas social e política do estado do Paraná. Foram feitas críticas às instituições mantenedoras dos periódicos, à burocracia, além da menção ao desafio de publicar um periódico de Ciência Humanas com pontualidade em meio à greve e à falta de apoio de editais de financiamento do CNPq/Capes e da Fundação Araucária, do Paraná (Costa, 2015). O editor relata a experiência negativa com a descontinuidade da indexação: "Certamente que tal decisão nos causou um grande impacto, mesclando frustração, decepção, desânimo e até um certo sentimento de culpa, embora nossa consciência nos mostrasse que fizemos o possível e o impossível para cumprir o critério de pontualidade" (Costa, 2015, p. 151). O texto do editor é um exemplo que retrata o quão importante é a indexação na SciELO para a comunidade acadêmica brasileira. Constatou-se que em 2019 Psicologia em Estudo voltou a ser indexado na Coleção SciELO Brasil. Os dados coletados, porém, referem-se a julho de 2018, por isso o título foi analisado como descontinuado na base.

Mueller (2011) analisa as fontes externas de financiamento dos periódicos indexados na SciELO e destaca que o CNPq/Capes auxilia 76,68\% dos títulos, um volume que denota a dependência de recursos estatais para a manutenção das publicações. Em segundo lugar estão as fundações de amparo estaduais (11,39\%), sendo encontradas somente três fundações, entre elas a Fundação Araucária. 
O terceiro motivo identificado foi ser "Contra a política da SciELO de internacionalização", expresso pelo periódico Scientiae Studia. Seus editores manifestaram o desacordo em relação às políticas da SciELO de internacionalização. Dedicado aos estudos da história, sociologia e filosofia da ciência e da tecnologia, seus editores criticaram as diretrizes da SciELO em relação à padronização dos periódicos, ao uso de sistema de submissão de manuscritos ligado a editoras comerciais e à necessidade de aumentar a publicação em língua inglesa, visto que o público alvo do título se compõe, principalmente, de pesquisadores da América Latina, Portugal e Espanha (Mariconda, 2015; Mariconda; Caponi, 2016).

Pires et al. (2020) explicam que há forte oposição nas áreas de humanidades e ciências sociais ao uso de análises bibliométricas elaboradas por bases como Web of Science e Scopus, por exemplo. Há, também, particularidades nas práticas de comunicação científica, como o interesse pela publicação em língua vernácula. Nesse sentido, publicar em inglês representaria a perda da "razão de ser" dos trabalhos (Pires et al., 2020, p. 11).

É fato que a SciELO estabelece parâmetros distintos de publicação para cada área do saber, indicando conhecimento das especificidades que caracterizam os periódicos indexados. Enquanto os títulos das Biológicas, por exemplo, devem publicar minimamente $85 \%$ de seus manuscritos em inglês, nas Humanas e Sociais Aplicadas a proporção cai para 25\%, e para Linguística, Letras e Artes, 20\% (Scientific..., 2017). O idioma de publicação é uma característica fundamental de um periódico, com forte impacto na audiência, no recebimento de novos manuscritos e em investimentos de tradução e revisão, portanto, é compreensível que o tema seja objeto de debate.

Por falta de editoriais com informações objetivas, não foi possível identificar os motivos da descontinuidade da indexação em 17 publicações, o que mostra a necessidade de ampliar a transparência entre periódicos e comunidade científica nas diversas áreas do conhecimento.

\section{Conclusão}

A pesquisa analisou as motivações que levaram 75 periódicos da Coleção SciELO a ter a indexação descontinuada. Observou-se que o período em que ocorre a desindexação coincide com mudanças importantes nas trajetórias da maioria dessas publicações. Das 75 publicações analisadas, que representam 21\% do total da base, 38 (51\%) permaneceram na SciELO mudando de título, indicando que a maioria mostrou capacidade de acompanhar as exigências tecnológicas e editoriais propostas pela base, que refletem padrões da comunicação científica internacional.

Identificaram-se cinco motivações na categoria terminado (40 títulos), em que os periódicos se mantiveram na base: Internacionalização e visibilidade internacional (62,5\%), Modernização (5,0\%), Mudança de entidade editora (5,0\%), Mudança de entidade editora e internacionalização (2,5\%) e Absorvido por outro periódico (2,5\%). Para nove títulos não foi possível identificar as motivações.

Identificaram-se três motivações na categoria indexação interrompida (35 títulos), em que os periódicos saíram da base: Internacionalização e/ou visibilidade internacional, parceria com editora comercial internacional (34,0\%), Atraso no lançamento de novos fascículos $(14,4 \%)$ e Contra a política da SciELO de internacionalização (2,9\%). Para 17 títulos não foi possível identificar as motivações.

No total,12 títulos tiveram a indexação descontinuada porque estabeleceram parcerias com as editoras comerciais internacionais Springer, Elsevier e APA, abandonando o OA e passando a cobrar subscrição, fenômeno conhecido como 'conversão contrária'. A análise dos editoriais sob a ótica da teoria de Bourdieu evidencia uma expectativa de obtenção de capital simbólico pela associação com editores internacionais de prestígio. Pode-se inferir que os objetivos desta parceria são a obtenção ou aumento de FI para o título, seguido de posicionamento em estratos superiores do Qualis Capes. 
As instituições editoras são peças fundamentais para a manutenção dos títulos, sendo necessário aprofundar o debate sobre a sustentabilidade das publicações com recursos das próprias instituições, como a cobrança de taxas aos autores e outras estratégias. As entidades públicas dedicadas à ciência brasileira não contribuem satisfatoriamente para garantir essa sustentabilidade, na medida em que não alocam com regularidade recursos para a manutenção dos periódicos científicos, criando entraves ao desenvolvimento sustentável do acesso livre.

O grande número de motivos não identificados pela falta de editoriais aponta para a necessidade de ampliar a transparência na gestão dos periódicos. Se o título é o canal de comunicação científica de uma área do conhecimento, que depende e conta com a periodicidade dos fascículos para publicar seus trabalhos, é essencial que a entidade publicadora se responsabilize não só pela sustentabilidade, mas também pela ampla divulgação das principais mudanças que ocorrem no periódico. Além disso, a história do periódico constitui inclusive parte da memória da ciência e por isso precisa ser registrada e preservada.

Este trabalho identificou linhas de investigação que merecem atenção: a importância dos editoriais como registro das mudanças nos periódicos, pois os documentos, quando encontrados, apresentavam justificativas superficiais e pouco fundamentadas; a necessidade de estudo aprofundado das parcerias estabelecidas entre as editoras comerciais, as sociedades científicas e as universidades responsáveis pela gestão editorial dos periódicos.

\section{Colaboradores}

J. P. S. PEREIRA contribuiu com o desenho, análise, interpretação dos dados, redação e revisão do texto. R. S. RODRIGUES contribuiu com o desenho, interpretação dos dados e revisão do texto. S. M. SANTOS contribuiu com a redação, interpretação dos dados e revisão do texto.

\section{Referências}

Abdalla, D. Editorial. Revista Brasileira de Ciências Farmacêuticas, v. 44, n. 1, p. 1-2, 2008. Doi: http://dx.doi.org/10.1590/ S1516-93322008000100001.

Associação Brasileira de Normas Técnicas. NBR 6021: publicação periódica técnica e/ou científica. Rio de Janeiro: ABNT, 2016.

Advertência. Boletim do Instituto Oceanográfico, v. 3, n. 1-2, p. 3-4, 1952. Doi: http://dx.doi.org/10.1590/S0373-5524 1952000100001.

Alperin, J. P.; Fischman, G. E.; Willinsky, J. Open access and scholarly publishing in Latin America: ten flavours and a few reflections. Liinc em Revista, v. 4, n. 2, p. 172-185, 2008. Doi: https://doi.org/10.18617/liinc.v4i2.269.

Appel, A. L; Albagli, S. The adoption of Article Processing Charges as a business model by Brazilian Open Access journals. Transinformação, v. 31, e180045, 2019. DOl: https:// doi.org/10.1590/2318-0889201931e180045.

Babini, D. Acceso abierto a la producción científica de América Latina y el Caribe: identificación de principales instituciones para estrategias de integración regional. Revista Iberoamericana de Ciencia, Tecnología y Sociedad, v. 6, n. 17, p. 1-24, 2011. Disponible en: http://www.revistacts.net/ volumen-6-numero-17/101-articulos/390-acceso-abiertoa-la-produccion-cientifica-de-america-latina-y-el-caribeidentificacion-de-principales-instituciones-para-estrategiasde-integracion-regional. Acceso en: 19 enero, 2020.
Bardin, L. Análise de conteúdo. Lisboa: Edições 70, 1979.

Behlau, M. Editorial. Jornal da Sociedade Brasileira de Fonoaudiologia, v. 24, n. 4, p. 5-7, 2012. Doi: http://dx.doi. org/10.1590/S2179-64912012000400001.

Beigel, F. Publishing from the periphery: structural heterogeneity and segmented circuits: the evaluation of scientific publications for tenure in argentina's CONICET. Current Sociology, v. 62, n. 5, p. 743-765, 2014. Doi: https://doi. org/10.1177/0011392114533977.

Bourdieu, P. O campo científico. In: Bourdieu, P. Pierre Bourdieu: sociologia. São Paulo: Ática, 1983. p. 122-155.

Bourdieu, P. Os usos sociais da ciência: por uma sociologia clínica do campo científico. São Paulo: Editora Unesp, 2004.

Bressan-Smith, R. Introducing the theoretical and experimental plant physiology: the new official journal of the Brazilian Society of Plant Physiology. Theoretical and Experimental Plant Physiology, v. 25, n. 1, p. 1, 2013. Doi: http:// dx.doi.org/10.1590/S2197-00252013000100001.

Bueno, B. O. Editorial. Educação e Pesquisa, v. 25, n. 2, p. 7-8, 1999. Doi: http://dx.doi.org/10.1590/S1517-97021999000200001.

Camargo, P. R. et al. The Brazilian Journal of Physical Therapy is now published by Elsevier: a step forward. Brazilian Journal of Physical Therapy, v. 20, n. 6, p. 493, 2016. Doi: http://dx.doi. org/10.1590/bjpt-rbf.2014.0196. 
Chaves, A. 2015: ano novo, RBCI nova! Revista Brasileira de Cardiologia Invasiva, v. 22, n. 4, p. 313, 2014. Doi: http://dx.doi. org/10.1590/0104-1843000000051.

Chaves, A. RBCl versão 2015. Revista Brasileira de Cardiologia Invasiva, v. 23, n. 1, p. 1, 2015. Disponível em: http://www.rbci. org.br/pt/rbci-versao-2015/articulo/S010418431500020X/. Acesso em: 21 dez. 2018.

Cômsoli, F. Editorial. Neotropical Entomology, v. 41, n. 1, 2012. Doi: https://doi.org/10.1007/s13744-012-0022-3.

Conselho Nacional de Desenvolvimento Científico e Tecnológico. Chamada CNPq no 19/2019: programa editorial. Brasília: CNPq, 2019. Disponível em: http://cnpq.br/chamadaspublicas?p_p_id=resultadosportlet_WAR_resultadoscnpqpo rtlet_INSTANCE_OZaM\&filtro=abertas\&detalha=chamada Divulgada\&idDivulgacao=8942. Acesso em: 16 nov. 2019.

Coordenação de Aperfeiçoamento de Pessoal de Nível Superior. Relatório do Qualis periódicos: área 40 - História. Brasília: Capes, 2019a. Disponível em: https://capes.gov. br/images/Relatorio_qualis_periodicos_referencia_2019/ Relatorio_qualis_historia.pdf. Acesso em: 30 jun. 2020.

Coordenação de Aperfeiçoamento de Pessoal de Nível Superior. Relatório do Qualis periódicos: área 38 - Educação. Brasília: Capes, 2019b. Disponível em: http://capes.gov. br/images/Relatorio_qualis_periodicos_referencia_2019/ Relatorio_qualis_educacao.pdf. Acesso em: 30 jun. $20 \overline{20}$.

Coordenação de Aperfeiçoamento de Pessoal de Nível Superior. Relatório do Qualis periódicos: área 35 - Antropologia e Arqueologia. Brasília: Capes, 2019c. Disponível em: https://www.capes.gov.br/images/Relatorio_qualis_periodicos_ referencia_2019/Relatorio_qualis_antropologia.pdf. Acesso em: 30 jun. 2020.

Coordenação de Aperfeiçoamento de Pessoal de Nível Superior. Relatório do Qualis periódicos: área 15 - Medicina I. Brasília: Capes, 2019d. Disponível em: https://capes.gov. br/images/Relatorio_qualis_periodicos_referencia_2019/ Relatorio_qualis_med1.pdf. Acesso em: 30 jun. 2020.

Costa, M. P.; Leite, F. C. L. Open access in the world and Latin America: a review since the Budapest Open Access Initiative. TransInformação, v. 28, n. 1, p. 33-45, 2016. Doi: http://dx.doi. org/10.1590/2318-08892016002800003.

Costa, P. J. Editorial. Psicologia em Estudo, v. 20, n. 2, p. 151, 2015. Doi: https://doi.org/10.4025/psicolestud.v20i2.30278.

Csomós, G. A spatial scientometric analysis of the publication output of cities worldwide. Journal of Informetrics, v. 12, n. 2, p. 547-566, 2018. Doi: https://doi.org/10.1016/j.joi.2018.05.003.

Editorial Note. Brazilian Journal of Biology, v. 62, n. 1, 2002. Doi: http://dx.doi.org/10.1590/S1519-69842002000100001.

Fachin, G. R. B.; Medeiros, G. M.; Rados, G. J. V. Padronização de periódicos científicos on-line da área de Biblioteconomia e Ciência da Informação: adequação às normas ISO. Revista ACB: Biblioteconomia em Santa Catarina, v. 13, n. 2, p. 415-438, 2008. Disponível em: https://revistaacb.emnuvens.com.br/ racb/article/view/539. Acesso em: 10 ago. 2019.

Fernandes, G. A. A. L.; Manchini, L. How QUALIS CAPES influences Brazilian academic production? A stimulus or a barrier for advancement? Brazilian Journal of Political Economy, v. 39, n. 2, p. 285-305, 2019. Doi: https://doi.org/10.1590/010131572019-3006.
Ferreira, E. I. Celebrating the 70th Anniversary with increased international visibility! Brazilian Journal of Pharmaceutical Sciences, v. 45, n. 1, 2009. Doi: http://dx.doi.org/10.1590/ S1984-82502009000100001.

Fonseca Junior, W. C. Análise de conteúdo. In: Duarte, J.; Barros, A. Métodos e técnicas de pesquisa em comunicação. 2. ed. São Paulo: Atlas, 2012.

Gingras, Y. The transformation of the scientific paper: from knowledge to accounting unit. In: Biagioli, M.; Lippman, A. Gaming the metrics: misconduct and manipulation in academic research. Boston: MIT Press, 2020. Doi: https://doi. org/10.7551/mitpress/11087.001.0001.

Guédon, J. C. El acceso abierto y la división entre ciencia "principal" y "periférica". Crítica y Emancipación, v. 3, n. 6, p. 135-180, 2011. Disponible em: http://eprints.rclis.org/17570/. Acceso en: 25 marzo, 2018.

International Organization for Standardization. 150 8: information and documentation: presentation and identification of periodicals. Geneva: ISO, 2019.

Jotz, G. P.; Bittencourt, A. International archives of otorhinolaryngology and thieme medical publishers. International Archives of Otorhinolaryngology, v. 17, n. 3, p. 236, 2013. Doi: http://dx.doi.org/10.7162/S1809-977720130003 00001.

Kapczinski, F. et al. New title: Trends in Psychiatry and Psychotherapy. Trends in Psychiatry and Psychotherapy, v. 33, n. 3, p. 133-134, 2011a. Doi: http://dx.doi.org/10.1590/S223760892011000300002.

Kapczinski, F. et al. Novas tendências da Revista de Psiquiatria do Rio Grande do Sul. Revista de Psiquiatria do Rio Grande do Sul, v. 33, n. 2, p. 69, 2011 b. Doi: http://dx.doi.org/10.1590/ S0101-81082011000200001.

Krauskopf, E. An analysis of discontinued journals by Scopus. Scientometrics, v. 16, n. 3, p.1805-1815, 2018. Doi: https://doiorg.ez46.periodicos.capes.gov.br/10.1007/s11192-018-2808-5.

Landeira-Fernandez, J. et al. Looking to the future: the American Psychological Association is the new publisher of Psychology \& Neuroscience. Psychology and Neuroscience, v. 8, n. 1, p. 1-3, 2015. Doi: http://dx.doi.org/10.1037/pne0000012.

Larivière, V.; Lozano, G. A.; Gingras, Y. Are elite journals declining? Journal of the Association for Information Science and Technology, v. 65, n. 4, p. 649-655, 2014.

Lin, S.; Zhan, L. International publishing partnerships for China's English language journals and financial security. Learning Publishing, v. 29, n. 3, p. 145-153, 2016. Doi: https:// doi.org/10.1002/leap.1029.

Mariconda, P. R. Editorial. Scientiae Studia, v. 13, n. 4, p. 727-730, 2015. Doi: http://dx.doi.org/10.1590/S1678-316620150004 00001.

Mariconda, P. R.; Caponi, G. Editorial. Scientiae Studia, v. 14, n. 1, p. 7-8, 2016. Doi: http://dx.doi.org/10.1590/S1678-3166 2016000100001.

Martinez, S. S. Editorial. Neotropical Entomology, v. 30, n. 1, 2001. Doi: https://doi.org/10.1590/S1519-566X2001000100001.

Matthias L.; Jahn N.; Laakso, M. The two-way street of open access journal publishing: flip it and reverse it. Publications, v. 7, n. 2, p. 29, 2019. Doi: https://doi.org/10.3390/publications 7020023 
Merton, R. Ensaios de sociologia da ciência. São Paulo: Editora 34, 2013.

Mograbi, D. C. Psychology \& Neuroscience indicators in 2013: evidence of growth and internationalization. Psychology and Neuroscience, v. 7, n. 2, p. 61-63, 2014. Doi: https://doi. org/10.3922/j.psns.2014.022.

Moraes, M.; Nascimento, M. L. Editorial. Fractal: Revista de Psicologia, v. 20, n. 1, 2008. Doi: http://dx.doi.org/10.1590/ S1984-02922008000100001.

Moura Duarte, F. A. Editorial. Genetics and Molecular Biology, v. 21, n. 1, p. 1-2, 1998. Doi: http://dx.doi.org/10.1590/S141547571998000100001

Mueller, S. P. M. Produção e financiamento de periódicos científicos de acesso aberto: um estudo na base SciELO. In: Poblaciòn, D. A. et al. Revistas científicas: dos processos tradicionais às perspectivas alternativas de comunicação. Cotia: Ateliê Editorial, 2011. p. 201-230.

Mugnaini, R.; Digiampetri, L. A.; Mena-Chalco, J.P. Comunicação científica no Brasil (1998-2012): indexação, crescimento, fluxo e dispersão. Transinformação, v. 26, n. 3, p. 239-252, 2014. Doi: http://dx.doi.org/10.1590/0103-3786201400030002.

Negahdary, M. Identifying scientific high-quality journals and publishers. Publishing Research Quarterly, v. 33, n. 4, p. 456470, 2017. Doi: https://doi-org.ez46.periodicos.capes.gov. $\mathrm{br} / 10.1007 / \mathrm{s} 12109-017-9541-4$

Oliveira, M. C. F.; Breitman, K. Joint Letter from the Editor-inChief and the publications chair of the Brazilian computer society. Journal of the Brazilian Computer Society, v. 15, n. 4, 2009. Doi: http://dx.doi.org/10.1007/BF03194508.

Packer, A. L. A eclosão dos periódicos do Brasil e cenários para o seu porvir. Educação e Pesquisa, v. 40, n. 2, 2014a. Doi: http:// dx.doi.org/10.1590/S1517-97022014061860.

Packer, A. L. SciELO Citation Index no Web of Science [online]. Scielo em Perspectiva, 2014b. Available from: https://blog. scielo.org/blog/2014/02/28/scielo-citation-index-no-web-ofscience/\#.X4RLkNBKjIU. Acess on: Mar 23, 2018.

Packer, A. L.; Meneghini, R. O SciELO aos 15 anos: raison d'être, avanços e desafios para o futuro. In: Packer, R. L. et al. SCIELO: 15 anos de acesso aberto: um estudo analítico sobre acesso aberto e comunicação científica. Paris: Unesco, 2014. Disponível em: http://www.scielo.org/php/level.php?lang= pt\&component=56\&item=61. Acesso em: 15 maio 2017 .

Paiva Filho, A. M. Editorial. Boletim do Instituto Oceanográfico, v. 43, n. 2, 1995. Doi: http://dx.doi.org/10.1590/S0373-5524 1995000200001.

Passos, P. C. S. J. et al. Critérios de qualidade em periódicos científicos. Informação e Sociedade: Estudos, v. 28, n. 2, p. 209-226, 2018. Disponível em: file:///C:/Users/JOSPAU 1/ AppData/Local/Temp/39101-100358-1-PB.pdf. Acesso em: 18 ago. 2019.

Pereira, J. P. S.; Rodrigues, R. S.; Santos, S. M. Periódicos científicos com indexação descontinuada: a Coleção Scielo Brasil. Zenodo. Version 1. 2020. Doi: http://doi.org/10.5281/ zenodo.3636067.

Pfenning, L. H. et al. Fitopatologia brasileira torna-se um periódico internacional. Tropical Plant Pathology, v. 33, n. 1, p. 3, 2008. Doi: http://dx.doi.org/10.1590/\$1982-56762008 000100001

Pires, A. S. et al. Implicações do sistema de classificação de periódicos Qualis em práticas de publicação no Brasil entre 2007 e 2016. Arquivos Analíticos de Políticas Educativas, v. 28, n. 25, p. 1-25, 2020. Doi: https://doi.org/10.14507/epaa.28.4353.

Pozzobon, A. P. B.; Camargo, G. A. A participação de editoras privadas em periódicos científicos brasileiros: uma visão sobre o gerenciamento privado de publicações acadêmicas. Perspectivas em Gestão e Conhecimento, v. 9, n. 2, p. 225-244, 2019. Doi: http://dx.doi.org/10.21714/2236417X2019v9n2p225.

Reichardt, K. Nota do editor. Anais da Escola Superior de Agricultura Luiz de Queiroz, v. 48, 1991. Doi: http://dx.doi. org/10.1590/S0071-12761991000100001.

Reichardt, K. Nota do editor. Scientia Agricola, v. 49, 1992. Doi: http://dx.doi.org/10.1590/S0103-90161992000400001.

Rocha-e-Silva, M. No fascículo de fevereiro 2005 de CLINICS. Clinics, v. 60, n. 1, p. 1-3, 2005. Doi: http://dx.doi.org/10.1590/ S1807-59322005000100001.

Rodrigues, R. S.; Fachin, G. R. B. Portal de periódicos científicos: um trabalho multidisciplinar. Transinformação, v. 22, n. 1, p. 33-45, 2010. Doi: http://dx.doi.org/10.1590/S0103-37862010 000100003.

Salatino, A. Editorial. Brazilian Journal of Botany, v. 35, n. 4, 2012. Doi: http://dx.doi.org/10.1590/S0100-84042012000400002.

Santos, S. M.; Mugnaini, R. Comunicação científica em periódicos e a internacionalização das ciências brasileiras. In: Carneiro, F. F. B.; Ferreira, Neto, A.; Santos, W. (org.). A comunicação científica em periódicos. Curitiba: Appris, 2019. p. 73-94.

Schifini, L. R. C.; Rodrigues, R. S. Política de avaliação de periódicos nas áreas de medicina: impactos sobre a produção editorial brasileira. Perspectivas em Ciência da Informação, v. 24, n. 4, p. 78-111, 2019. Doi: https://doi.org/10.1590/1981$5344 / 3745$.

Scientific Electronic Library Online. Critérios, política e procedimentos para a admissão e apermanência de periódicos científicos na Coleção SciELO Brasil. São Paulo: Scielo, 2014. Disponível em: https://wp.scielo.org/wp-content/uploads/20140900Criterios-SciELO-Brasil.pdf. Acesso em: 18 jun. 2020

Scientific Electronic Library Online. Critérios, política e procedimentos para a admissão e a permanência de periódicos científicos na Coleção SciELO Brasil. São Paulo: Scielo, 2017.

Silva, I. N. Editorial: new Journal for Publishing Relevant Research in Control, Automation and Electrical Systems. Journal of Control, Automation and Electrical Systems, v. 24, n. 1-2, p. 1-2, 2013. Doi: https://doi.org/10.1007/s40313-0130024-3.

$\mathrm{Xu}$, J. et al. Internationalization of China's English-language academic journals: an overview and three approaches. Learned Publishing, v. 32, n. 2, p. 113-125, 2019. Doi: https:// doi.org/10.1002/leap.1198. 